\title{
La communication entre médecin traitant et médecin-conseil
}

\author{
Pour le médecin-conseil et le médecin traitant, la priorité est donnée à un traite- \\ ment médical adéquat. L'appréciation médicale pour la décision de prise en charge \\ par l'assureur d'une prestation à fournir ou fournie sert le plus souvent des intérêts \\ communs.
}

Les médecins traitants (MT) dénoncent la qualité souvent insuffisante des décisions négatives des assureurs et l'accessibilité difficile du médecin-conseil (MC) compétent pour un échange professionnel direct. Alors que le travail des médecins-conseils (MC) peut être compliqué par des demandes de garantie de prise en charge des coûts lacunaires des MT, ces derniers se plaignent de questions inutiles et insuffisamment compétentes des assureurs.

Afin d'améliorer la compréhension mutuelle, les comités de direction de la SSMC et de la CCM ont adopté les recommandations ci-dessous en février 2017. Ces dernières devraient avoir un effet favorable sur le travail des MT et des MC.

\section{Demandes de rapport des assureurs}

Les assureurs demandent des rapports aux médecins traitants, en général via leurs services prestations. Les assureurs sont autonomes en la matière. Ils décident dans quels cas ils lancent des demandes, afin de satisfaire à leur obligation de vérification des prestations. Les rapports demandés sont souvent ressentis comme inutiles, voire chicaniers, par les MT.

Les demandes de rapport ne sont pas rédigées par le MC, mais habituellement par le service prestations de l'assureur, et contiennent souvent des questions standardisées.

Conclusion: Si le médecin traitant estime que la demande de rapport reçue est inopportune, il doit donc s'adresser exclusivement au service demandeur de l'assurance et non au service du médecin-conseil (SMC).

\section{Décisions de prestation négatives}

Ces décisions sont prises exclusivement par le service prestations de l'assurance, par correspondance en par- tie individuelle, en partie normée. Savoir dans quelle mesure une décision négative est présentée de façon transparente et si on peut en déduire si et de quelle manière le $\mathrm{MC}$ y a participé, est un autre problème. Conformément à l'art. 57 LAMal, le MC conseille l'assureur. Il n'a aucune compétence de décision. Un exemple typique de l'activité quotidienne du médecin-conseil est de conseiller l'assureur au sujet de la concrétisation d'une limitation. Si les critères de limitation ne sont pas remplis, le MC doit le signaler à l'assureur. Si un tel cas est évoqué dans un refus, il est indiqué comme ayant été décidé «après consultation du médecinconseil», ce qui est imprécis. Il serait plus précis de montrer la limitation, d'indiquer le conseil du MC et la décision du service prestations de refuser l'obligation de prestations qui en découle. Une réduction à la formulation "refus après consultation du médecinconseil» entraîne des conclusions erronées.

Avant de demander à consulter le MC, le MT doit s'assurer des raisons du refus. Si elles sont de nature formelle, une consultation du MC n'apporte rien en vue d'une modification souhaitée de la décision de l'assureur. Le refus d'une prestation est un processus formel. Avant d'intervenir en cas de désaccord ou d'incompréhension, il est recommandé de se faire une idée des raisons exactes du refus. Une communication transparente de la part du service prestations est ici primordiale.

L'interlocuteur principal est l'assureur puisque c'est lui qui prend la décision de prestation.

Par contre, si le refus d'une prestation ou d'une demande de garantie de prise en charge des coûts fondé sur l'évaluation EAE (efficacité, adéquation, économicité en comparaison thérapeutique transversale) repose clairement sur un jugement du MC, alors nous recommandons qu'une concertation avec le MC compétent soit rendue possible. L'accessibilité dans un délai utile du MC comme du MT doit être assurée. 
Conclusion: Une consultation mutuelle doit être rendue possible, dans la mesure où une recommandation négative du MC est à l'origine du refus. Par contre, dans le cas où la raison du refus est une norme de prestation d'ordre supérieur, la consultation du MC n'a aucun sens puisque dans ce cas l'assureur ne peut pas accorder de prestation pour des raisons formelles. L'assureur doit alors exposer clairement les raisons formelles.

\section{Droit à l'information de la part du médecin traitant}

Un tel droit n'existe pas, ni vis-à-vis de l'administration de l'assurance, ni vis-à-vis du MC, excepté quand le médecin-conseil examine lui-même la personne assurée (art. 57 al. 6 LAMal).

La personne assurée elle-même a par contre droit à l'information et à l'accès au dossier. Elle seule a aussi qualité pour recourir. Le médecin traitant peut se faire autoriser par la personne assurée à demander des informations directement auprès de l'assureur.

Conclusion: Le MT n'a aucun droit à l'information ou à l'obtention d'une décision susceptible de recours, sauf si la personne assurée l’y autorise.

\section{Aperçu des principaux points}

- Les rapports sont en règle générale demandés par l'administration de l'assurance. Les questions à ce sujet doivent être adressées à cet organisme.

- En cas de refus de prestation, il faut d'abord déterminer quelles en sont les raisons, afin de procéder de manière plus ciblée aux demandes de précisions.
Pour cela, les refus doivent être justifiés de manière transparente. Une concertation avec le MC n'a pas de sens si la raison du refus vient d'une ordonnance ou d'une loi.

- Le MC conseille l'assureur, il n'a aucune compétence de décision. Si une norme, comme par exemple une limitation ou un tarif manquant, détermine le refus, le MC n'a pas compétence pour ordonner la prise en charge des coûts à l'administration de la caisse, même en cas de vraisemblance médicale. Le traitement est une question de médecine, la prise en charge de la prestation est une question de droit applicable.

- Le MT n'a aucun droit légal à la concertation avec le MC. Par contre, nous recommandons qu'en cas de besoin une consultation soit rendue possible, si un refus a été clairement décidé sur la base de l'évaluation d'un médecin-conseil.

- Lors des demandes de rapport de la part de l'assureur, une enveloppe réponse au service du médecinconseil est généralement jointe. Le rapport parvient donc à ce dernier.

- Les demandes de garantie de prise en charge des coûts doivent être formulées de manière concise et complète. Les demandes de précisions ou refus inutiles peuvent ainsi être réduits. Elles doivent généralement mentionner le numéro de téléphone direct et l'adresse électronique du médecin demandeur. Les formulaires de garantie de prise en charge des coûts, que l'on peut trouver pour les différentes prestations sur le site Internet de la SSMC, facilitent le travail du MT comme du MC. 


\title{
Explications relatives aux recomman- dations de la CCM et de la SSMC
}

\author{
Peter Wiedersheima ${ }^{a}$, Jürg Zollikofer ${ }^{b}$ \\ ${ }^{a}$ Coprésident KKA-CCM; ${ }^{\text {b }}$ Président SGV/SSMC
}

\section{Contexte}

Ces recommandations devraient améliorer la communication entre les médecins traitants (MT) et les médecins-conseils (MC).

Les MT se plaignent des nombreuses questions standardisées et souvent peu qualifiées des assureurs ainsi que de l'insuffisance de la justification, du manque de qualité des décisions négatives et de l'inaccessibilité du MC compétent dans de tels cas. Le manque d'accessibilité repose en règle générale sur un refus de l'assureur de communiquer le nom du MC impliqué dans une décision négative.

De la part des MC, les motifs de rejet éventuel les plus fréquemment invoqués sont la qualité insuffisante des demandes de garantie de prise en charge des coûts et souvent aussi le manque de connaissance des faits et processus fixés par les lois et les ordonnances.

\section{Indépendance des médecins-conseils vis-à-vis de l'assureur}

Selon l'article 57 al. 5 LAMal et l'article 5 al. 1 de la Convention relative aux médecins-conseils, le MC est indépendant dans l'appréciation de questions techniques d'ordre médical. Il n'est lié par aucune instruction émise par l'assureur ou la société médicale en matière de technique médicale. Cf. http://www. medecins-conseils.ch/filesbon/sgv/conv_rel_aux_ medecins_conseils.pdf.

\section{Désignation des médecins-conseils}

La CCM critique le fait qu'en général la désignation des MC ne s'effectue plus conformément à la LAMal, la consultation de la société cantonale compétente par l'assureur-maladie est pourtant prévue par la loi et réglée à l'art. 57 LAMal, al. 1 et 2.

On ne peut que constater que ces dispositions légales ne sont plus observées. Du point de vue de la CCM, il s'agit là d'un abus qui devrait être poursuivi.

\section{Liste des médecins-conseils}

La demande pressante de la CCM pour que les adresses de tous les MC soient rendues publiques a entraîné de vives discussions au sein du groupe de travail. La SSMC émet des réserves claires à l'égard de la publication d'une telle liste. $90 \%$ des assurés sont clients de l'une des 12 grandes compagnies d'assurance. Ces dernières comptent jusqu'à 2 douzaines de $\mathrm{MC}$ dans leurs services. Par conséquent une telle liste ne sert pas à grandchose. En outre, la publication de l'adresse privée des MC n'est pas licite pour des raisons de protection des données. Le respect de la protection des données est l'affaire des assureurs, sous contrôle de l'OFSP. Seuls les assureurs pourraient publier une telle liste, et non la SSMC, car elle ne dispose ni de l'autorisation ni des informations nécessaires.

La CCM ne comprend pas cette argumentation. On ne peut vérifier si les MC possèdent les qualifications nécessaires selon la LAMal et la Convention MC que si les MC sont connus. A quoi sert le droit d'opposition d'une société cantonale de médecine mentionné dans la LAMal dans la situation actuelle où le nom n'est même pas connu? Comment peut-on p. ex. respecter l'art. 42 LAMal, al. 5, qui stipule que le fournisseur de prestations est fondé lorsque les circonstances l'exigent, ou astreint dans tous les cas si l'assuré le demande, à ne fournir les indications d'ordre médical qu'au médecin-conseil de l'assureur, conformément à l'art. 57?

\section{Accessibilité des médecins-conseils}

Pour la CCM comme pour la SSMC, il est incontestable que dans les cas où le refus de l'assureur s'appuie clairement sur une appréciation du MC, celui-ci doit être disponible pour une discussion avec le MT, par téléphone ou par courriel. Mais il est essentiel qu'avant une intervention le MT vérifie qui est vraiment à l'origine du refus fâcheux pour lui: le MC ou le service prestations de l'assureur. Cela justement est souvent difficile 
selon la CCM, car les communications de l'assureur-maladie manquent souvent de transparence et de clarté.

\section{«Pouvoir» des médecins-conseils}

Selon l'art. 57 al. 4 LAMal, le médecin-conseil donne son avis à l'assureur sur des questions médicales ainsi que sur des questions relatives à la rémunération et à l'application des tarifs. Il examine en particulier si les conditions de prise en charge d'une prestation sont remplies. Les MC n'ont aucun pouvoir de décision, juste un droit de recommandation.

\section{Compétences professionnelles des médecins-conseils}

Les MC sont en majorité des spécialistes en médecine interne générale, mais en partie aussi d'autres disciplines de médecine interne ou chirurgicales et des psychiatres. Ce ne sont pas des contrôleurs techniques des différents spécialistes. Il faut bien plus les aborder comme des médecins de premier recours qui doivent pouvoir expliquer leurs jugements et leurs plans au spécialiste. Il est donc très important que les MT rédigent des rapports concis contenant l'essentiel.

\section{Demande de garantie de prise en charge des coûts}

Dans l'intérêt personnel des MT, la demande doit être complète, et donc exposer les faits nécessaires au MC chargé de l'évaluation de la demande. Les diagnostics ne sont en soi pas toujours suffisants. Tandis que p. ex. la clinique joue un rôle important pour une utilisation off-label en oncologie, ce sont la plupart du temps les déficits fonctionnels (déficits dans l'AVQ) qui sont intéressants pour les demandes de séjours de réadaptation. NB: la qualité de l'appréciation du MC ne saurait être meilleure que la qualité de la demande de garantie de prise en charge. Sont en outre utiles selon la SSMC: le numéro de téléphone direct et l'adresse électronique du médecin demandeur. La CCM ne peut qu'approuver, avec réciprocité.

\section{Justification des décisions négatives des assureurs}

CCM et SSMC s'accordent sur le fait que les assureurs doivent justifier les refus de façon claire par souci de transparence. Si le MC n'est pas mentionné, sa recommandation en texte clair doit être jointe à la lettre de refus. Nous rejetons les formulations telles que «après consultation du MC» qui sont inacceptables, car on ne voit pas alors quels sont les termes de la recommandation. Il se peut tout à fait que l'on ait juste demandé au MC si les conditions d'une limitation étaient remplies. Si ce sont des lois ou des ordonnances qui excluent la prise en charge des coûts par l'assureur, elles doivent être clairement citées. Ce sont souvent des motifs législatifs ou purement administratifs, et non médicaux, qui jouent le rôle déterminant.

\section{Dispositions}

Seuls les patients ont le droit en cas de refus de demander une décision susceptible de recours parce qu'eux seuls ont un lien contractuel avec l'assureur. Le MT peut évidemment aussi, avec l'autorisation nécessaire, demander une décision susceptible de recours au nom de l'assuré.

\section{Rapports}

En règle générale, les demandes de rapports ne viennent pas du MC mais du service prestations de l'assureur, même si la réponse du MT est renvoyée dans une enveloppe adressée au médecin-conseil. Outre ces demandes d'assurance, des demandes de routine imposées sont aussi présentées, comme p. ex. lors d'une révision des rentes ou concernant la capacité de travail. La routine administrative peut et doit être allégée quand les rapporteurs peuvent fournir l'information nécessaire à moindre effort.

\section{Quintessence}

Comme toujours on peut constater que le système fonctionne bien dans la grande majorité des cas. Une bonne communication d'égal à égal entre les médecins traitants et les médecins-conseils chargés de l'appréciation/recommandation, ainsi que la connaissance mutuelle des droits, obligations et possibilités y participent.

\section{Membres du GT CCM-SSMC}

\author{
Markus Bonelli \\ Fiorenzo Caranzano \\ Rudolf Häuptle \\ Valentin Rehli \\ Peter Wiedersheim \\ Barbara Zinggeler \\ Jürg Zollikofer
}

